\title{
INTERNAL WAVE DIFFRACTION BY A STRIP OF AN ELASTIC PLATE ON THE SURFACE OF A STRATIFIED FLUID
}

\author{
P. DOLAI \\ Department of Mathematics \\ Prasanna Deb Women's College \\ Jalpaiguri-735101 \\ West Bengal, INDIA \\ D.P. DOLAI \\ River Research Institute \\ Govt. of West Bengal \\ 11A, Mirza Ghalib Stret \\ Kolkata-700087, INDIA \\ E-mail: dyutidolai@yahoo.co.in
}

\begin{abstract}
The problem of internal wave diffraction by a strip of an elastic plate of finite width present on the surface of an exponentially stratified liquid is investigated in this paper. Assuming linear theory, the problem is formulated in terms of a function related to the stream function describing the motion in the liquid. The related boundary value problem involves a hyperbolic type partial differential equation (PDE), known as the Klein Gordon equation. The method of Wiener-Hopf is utilized in the mathematical analysis to a slightly generalized boundary value problem (BVP) by introducing a small parameter, and the problem is solved approximately for large width of the plate. In the final results, this small parameter is made to tend to zero. The diffracted field is obtained in terms of integrals, which are then evaluated asymptotically in different regions for a large distance from the edges of the plate and the results are interpreted physically.
\end{abstract}

Key words: stratified fluid, Klein-Gordon equation, Wiener-Hopf technique, steepest descent method.

\section{Introduction}

If a part of the surface of a deep liquid is covered by an inertial surface composed of a thin but uniform distribution of non-interacting floating particles (e.g., broken ice, floating mat) while the remaining part is free, the surface boundary condition becomes discontinuous in the sense that there are one condition on the free surface and another condition on the inertial surface. In the mathematical modeling of wave phenomena, the line separating the free surface and the inertial surface becomes a line of discontinuity. The scattering of surface waves traveling from the free surface region and normally or obliquely incident on the line separating the free surface and the inertial surface on a homogenous fluid are investigated in the literature by many researchers. Kanoria et al. (1999) investigated two mixed boundary value problems involving surface water wave in deep water (or interface wave in two superposed homogeneous liquids) arising due to one or two discontinuities in the surface (or interface) boundary conditions. In this problem, the governing partial differential equation is the Laplace equation which was generalized to Helmholtz's equation together with slightly different boundary conditions by introducing a complex parameter to facilitate the use of the Wiener-Hopf technique in the mathematical analysis. Ultimately, this parameter was made to tend to zero to obtain the solution of the original problems. Instead of a homogeneous liquid, there

\footnotetext{
* To whom correspondence should be addressed
} 
may be a stratified liquid in which the density varies exponentially along the vertical direction. Assuming linear theory and under Boussinesq approximation with constant Brunt - Vaisala frequency, the problem can be formulated as a boundary value problem involving the Klein-Gordon equation with discontinuous surface boundary condition. Gabov and Svesnikov (1982) investigated diffraction of two-dimensional steady-state internal waves described by the Klein-Gordon equation in an exponentially stratified incompressible liquid by the boundary of a solid half plane lying on the free surface modeled as an ice field on the surface of an infinitely deep ocean. In the mathematical analysis, they employed the Wiener-Hopf technique. Afterwards, several researchers investigated a number of diffraction problems by using the same technique. For example, Varlamov (1983; 1985) investigated internal wave diffraction by a semi-infinite horizontal wall present inside the liquid and by a semi-infinite elastic half plate present on the surface of the liquid. Gayen et al. (2006; 2007) considered the problems of water wave diffraction by a surface strip and water wave scattering by two sharp discontinuities in the surface boundary conditions. Dolai and Mandal (2007) considered the problem of internal wave scattering by the edge of a semi-infinite inertial surface partly covering an exponentially stratified incompressible liquid of infinite depth. The concerned boundary value problem was solved by the use of Wiener-Hopf technique and obtained an asymptotic form of scattered field for a large distance from the edge.

In the present paper, the problem of internal wave diffraction by a strip of an elastic plate present on the upper surface of an exponentially stratified liquid of infinite depth is investigated. To solve the problem, the Wiener-Hopf technique is employed after introducing a complex parameter, as well as by slightly generalizing the surface boundary conditions, the edge conditions and the infinity requirements and taking the strip width to be large. The diffracted field is obtained in terms of integrals which are evaluated asymptotically for large distances from the edges of the plate by the method of steepest descent and interpreted physically.

For completeness, derivation of the Klien-Gordon equation is shown here by considering twodimensional motion in a stratified liquid occupying the region $y \leq 0$ when at rest, wherein the $y$-axis is chosen vertically upwards so that the upper surface of the liquid at the rest position coincides with the plane $y=0$. In the unperturbed state the density of the liquid is assumed to be of the form $\rho_{0}(0) \exp (-\beta y),(\beta>0)$, where $\rho_{0}(0)$ is the density at the top of the liquid, $\rho_{0}(y)$ being the unperturbed density at depth $y$. Then the linearised equations of motion are given by

$$
\begin{aligned}
& \rho_{0}(y) \frac{\partial v_{x}}{\partial t}=-\frac{\partial p}{\partial x}, \\
& \rho_{0}(y) \frac{\partial v_{y}}{\partial t}=-\frac{\partial p}{\partial y}-\rho g, \\
& \frac{\partial \rho}{\partial t}+v_{y} \rho_{0}^{\prime}(y)=0,
\end{aligned}
$$

while the equation of continuity is

$$
\frac{\partial v_{x}}{\partial x}+\frac{\partial v_{y}}{\partial y}=0
$$

where $v_{x}$ and $v_{y}$ denote the velocity components along the $x$ and $y$ directions, respectively, $\rho$ denotes the perturbed density due to the motion in the liquid, $p$ denote the dynamic pressure.

If $\psi(x, y ; t)$ denotes the stream function describing the motion in the liquid, then 


$$
v_{x}=\frac{\partial \psi}{\partial y}, \quad v_{y}=-\frac{\partial \psi}{\partial x} .
$$

If we substitute

$$
\psi(x, y ; t)=\Theta(x, y ; t) \exp (\beta y),
$$

it is then found that Eqs (1.1) to (1.4), after using the Boussinesq approximation, ultimately produce the partial differential equation

$$
\frac{\partial}{\partial t^{2}}\left(\nabla^{2} \Theta-\beta^{2} \Theta\right)+\omega_{0}^{2} \frac{\partial^{2} \Theta}{\partial x^{2}}=0, \quad y \leq 0
$$

where $\nabla^{2}$ denotes the two-dimensional Laplacian and $\omega_{0}=(2 \beta g)^{1 / 2}$ is the so-called Brunt-Vaisala frequency.

For plane wave solutions of the PDE (1.7), we write $\Theta(x, y ; t)=\operatorname{Re}\{\Theta(x, y) \exp (-i \omega t)\}$, where $\Theta(x, y)$ is now a complex valued function, $\omega$ is the circular frequency. If $\exp \left(i k_{1} x+i k_{2} y-i \omega t\right)$ represents the plane wave solution of Eq.(1.7), then the dispersion relation is

$$
\omega^{2}=\frac{\omega_{0}^{2} k_{1}^{2}}{k_{1}^{2}+k_{2}^{2}+\beta^{2}} \text {. }
$$

The group velocity $V_{g}=\left(\frac{\partial \omega}{\partial k_{1}}, \frac{\partial \omega}{\partial k_{2}}\right)$ is then obtained as

$$
V_{g}=\frac{\omega_{0} \operatorname{sign}\left(k_{1}\right)}{\left(k_{1}^{2}+k_{2}^{2}+\beta^{2}\right)^{3 / 2}}\left(k_{2}^{2}+\beta^{2},-k_{1} k_{2}\right) .
$$

Thus the directions of the group velocity $V_{g}$ and the wave vectors $k=\left(k_{1}, k_{2}\right)$ do not coincide unless $k_{2}=0$. Also, as the direction of $V_{g}$ determines the direction of energy flow in the wave, the direction of wave propagation is to be taken as the direction of $V_{g}$ rather than that of $k$. Again, from the dispersion relation (1.8), it is ensured that plane wave type solutions are possible only when $\omega<\omega_{0}$ and this will be assumed all throughout here. Thus the complex valued function $\Theta(x, y)$ satisfies

$$
\frac{\partial^{2} \Theta}{\partial y^{2}}-\beta^{2} \Theta=\frac{1}{a^{2}} \frac{\partial^{2} \Theta}{\partial x^{2}}, \quad y \leq 0
$$

which is the Klein-Gordon equation, where

$$
\frac{1}{a^{2}}=\frac{\omega_{0}^{2}}{\omega^{2}}-1
$$




\section{Formulation of the problem}

Let an incompressible inviscid exponentially stratified liquid occupy the half space $y \leq 0$ when at rest and the plane $(y=0, x<0) \cup(y=0, x>l)$ be the rest position of the free surface while the plane $(y=0,0<x<l)$ be the rest position of the elastic plate with surface density $\sigma_{l}$. For two-dimensional motion in the liquid, the linearised conditions at the free surface and at the plate surface are

$$
\frac{g}{\omega^{2}} \frac{\partial^{2} \Theta}{\partial x^{2}}+\frac{\partial \Theta}{\partial y}+\beta \Theta=0 \quad \text { on } \quad y=0, \quad(x<0) \bigcup(x>l),
$$

and

$$
\frac{c}{\rho_{0} \omega^{2}} \frac{\partial^{2} \Theta}{\partial x^{2}}+\frac{\partial \Theta}{\partial y}+\beta \Theta=0 \quad \text { on } \quad y=0, \quad 0<x<l
$$

where $\rho_{0} \equiv \rho_{0}(0)$ and

$$
c=g \rho_{0}-\sigma_{1} \omega^{2} .
$$

We can assume that the constant $c$ is a positive quantity as long as $\omega<\omega_{0}$. This is due to the fact that under actual conditions concerning a stratified ocean, $\omega_{0}^{2} \approx 10^{-4} \mathrm{~Hz}^{2}$ and when the ocean is covered by a thin elastic plate of surface density $\sigma_{1}, \frac{g \rho_{0}}{\sigma_{l}} \approx 10 H z^{2}$ (cf. Varlamov (1983)). Thus $\omega_{0}^{2}<\frac{g \rho_{0}}{\sigma_{l}}$ and hence $c>0$ since $\omega<\omega_{0}$ has already been assumed.

Let from the region $x<0, y<0$, a plane wave field represented by $\phi_{0}(x, y)=\exp (-i b y+i k x)$ where $k^{2}=a^{2}\left(b^{2}+\beta^{2}\right)$ and $b, k$ are taken to be positive, propagate from infinity and be incident on the edge of the inertial surface separating the free surface. The total wave field $\Theta$ can be represented in the form

where

$$
\Theta(x, y)=\phi_{0}(x, y)+\phi_{1}(x, y)+\phi(x, y)
$$

with

$$
\phi_{1}(x, y)=\mathrm{R} \exp (i b y+i k x)
$$

$$
R=\frac{i b-\beta+\frac{g k^{2}}{\omega^{2}}}{i b+\beta-\frac{g k^{2}}{\omega^{2}}},
$$

so that it represents the wave reflected from the free surface and $\phi(x, y)$ is the diffracted field, which satisfies the boundary value problem described by the Klein-Gordon equation

$$
\frac{\partial^{2} \phi}{\partial y^{2}}-\beta^{2} \phi=\frac{1}{a^{2}} \frac{\partial^{2} \phi}{\partial x^{2}}, \quad y<0,
$$

and the boundary conditions 


$$
\begin{aligned}
& \frac{g}{\omega^{2}} \frac{\partial^{2} \phi}{\partial x^{2}}+\frac{\partial \phi}{\partial y}+\beta \phi=0 \quad \text { on } \quad y=0, \quad(x<0) \bigcup(x>l), \\
& \frac{\partial^{6} \phi}{\partial x^{6}}+\frac{c}{\lambda} \frac{\partial^{2} \phi}{\partial x^{2}}+\frac{\rho_{0} \omega^{2}}{\lambda}\left(\frac{\partial \phi}{\partial y}+\beta \phi\right)=A \exp (i k x) \quad \text { on } \quad y=0, \quad 0<x<l
\end{aligned}
$$

where $\lambda$ denotes the rigidity of the elastic plate and

$$
A=\frac{k^{2}\left(k^{2}-\frac{\sigma_{l} \omega^{2}}{\lambda}\right)}{1+R} .
$$

For the edge conditions, the derivative of $\phi$ upto the third order should be bounded at the edges and the higher derivatives at these points should have a singularity.

To apply the Wiener-Hopf technique for finding the solution for $\phi$, we assume that the constant $a$ occurring in the PDE (2.6) has a small positive imaginary part $\varepsilon$ so that the constant $k$ has a positive imaginary part $\delta(\varepsilon)=\left(b^{2}+\beta^{2}\right)^{1 / 2} \varepsilon$ which tends to zero as $\varepsilon \rightarrow 0+$.

Also $\phi$ satisfies the edge conditions

$$
\left|\frac{\partial^{4} \phi}{\partial x^{4}}\right|=\left|\frac{\partial^{5} \phi}{\partial x^{5}}\right|=\left|\frac{\partial^{6} \phi}{\partial x^{6}}\right| \leq \text { constant } r^{-1 / 2}
$$

where $r=x$ as $x \rightarrow 0+$ and $r=l-x$ as $x \rightarrow l-0$ on $y=0$, and the condition at infinity, as given by

$$
|\phi|+|\nabla \phi|+\cdots+\left|\frac{\partial^{6} \phi}{\partial x^{6}}\right| \leq \text { constant } \exp (-\chi(\varepsilon) r) \quad \text { as } \quad r=\left(x^{2}+y^{2}\right)^{1 / 2} \rightarrow \infty
$$

where $0<\chi(\varepsilon) \leq \min (\varepsilon \beta, \delta(\varepsilon))=\varepsilon \beta$ so that $\chi(\varepsilon) \rightarrow 0$ as $\varepsilon \rightarrow 0+$.

In the next section, the three-part Wiener-Hopf technique is applied to solve the generalized BVP satisfying the Klein-Gordon Eq.(2.6) involving the complex parameter $a$, the surface boundary conditions (2.7) and (2.8), and the edge conditions (2.9) and the infinity requirements (2.10).

\section{Solution of the problem}

Let $\Phi(\alpha, y)$ denote the Fourier transform of $\phi(x, y)$ defined by

$$
\Phi(\alpha, y)=\int_{-\infty}^{\infty} \phi(x, y) \exp (i \alpha x) d x
$$

where $\alpha=\sigma+i \tau, \sigma$ and $\tau$ being real. Then 


$$
\Phi(\alpha, y)=\Phi^{-}(\alpha, y)+\exp (i \alpha l) \Phi^{+}(\alpha, y)+\int_{0}^{l} \phi(x, y) \exp (i \alpha x) d x
$$

where,

$$
\Phi^{-}(\alpha, y)=\int_{-\infty}^{0} \phi(x, y) \exp (i \alpha x) d x, \quad \Phi^{+}(\alpha, y)=\int_{l}^{\infty} \phi(x, y) \exp (i \alpha(x-l)) d x .
$$

By using the condition (2.10) it is observed that $\Phi^{+}(\alpha, y)$ is regular in the upper half plane $\tau>-\chi(\varepsilon)$ and $\Phi^{-}(\alpha, y)$ is regular in the lower half plane $\tau<\chi(\varepsilon)$ of the complex $\alpha$-plane. Again by using the edge condition (2.9) along with the Abelian theorem (cf. Noble (1958)) we ensure that

$$
\left|\Phi^{ \pm}(\alpha, y)\right|=O\left(|\alpha|^{-1}\right) \quad \text { as } \quad|\alpha| \rightarrow \infty \quad \text { in } \quad \tau_{>}^{<} \mp \chi(\varepsilon) .
$$

To use the Wiener - Hopf procedure, the boundary conditions (2.7) and (2.8) are written in the forms

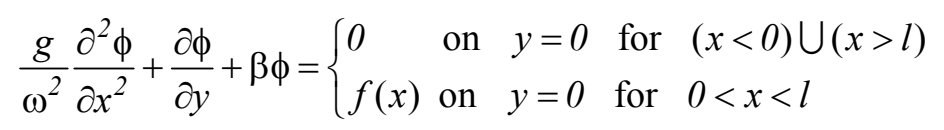

and

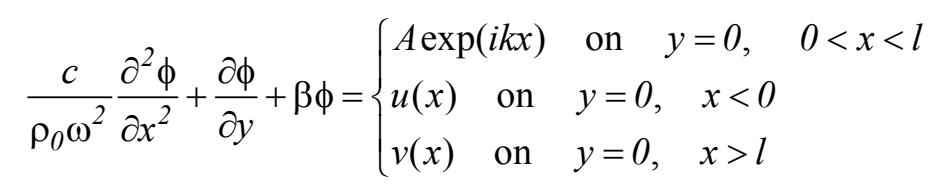

where $f(x)$ (for $0<x<l$ ), $u(x)$ (for $x<0$ ) and $v(x)$ (for $x>l$ ) are unknown functions having the behaviour at the points $x=0$ and $x=l$, as given by

$$
\begin{aligned}
& f(x)=O(1) \quad \text { as } \quad x \rightarrow 0+\quad \text { and } \quad x \rightarrow l- \\
& u(x)=O(1) \quad \text { as } \quad x \rightarrow 0- \\
& v(x)=O(1) \quad \text { as } \quad x \rightarrow l+.
\end{aligned}
$$

Now, the application of the Fourier transform technique to the PDE (2.6) produces the ordinary differential equation

$$
\frac{d^{2} \Phi}{d y^{2}}-\frac{\gamma^{2}(\alpha)}{a^{2}} \Phi=0, \quad y<0
$$

where $\gamma^{2}(\alpha)=\alpha^{2}-a^{2} \beta^{2}$ and we choose that branch of the function $\gamma(\alpha)=\left(\alpha^{2}-a^{2} \beta^{2}\right)^{1 / 2}$ for which $\gamma(0)=-i a \beta$ in the complex $\alpha$-plane cut along the line joining the points $-a \beta$ and $a \beta$ through infinity. A solution of Eq.(3.6) is

$$
\Phi(\alpha, y)=D(\alpha) \exp \left\{\frac{i \gamma(\alpha)}{a} y\right\}, \quad y<0
$$


where $D(\alpha)$ is an arbitrary function of $\alpha$, and is determined from the relations obtained by using the Fourier-transform on conditions (3.3) and (3.4) as given by

$$
\left(\beta-\frac{c \alpha^{2}}{\rho_{0} \omega^{2}}\right) \Phi(\alpha, 0)+\frac{d \Phi}{d y}(\alpha, 0)=U^{-}(\alpha)+V^{+}(\alpha) \exp (i \alpha l)+\frac{A}{i(\alpha+k)}\{\exp [i(\alpha+k) l]-1\}(3.8
$$

and

$$
\left(\beta-\frac{g \alpha^{2}}{\omega^{2}}\right) \Phi(\alpha, 0)+\frac{d \Phi}{d y}(\alpha, 0)=F(\alpha) .
$$

In Eqs (3.8) and (3.9) the three unknown functions $U^{-}(\alpha), V^{+}(\alpha)$ and $F(\alpha)$ are defined by

$$
\begin{aligned}
& U^{-}(\alpha)=\int_{-\infty}^{0} u(x) \exp (i \alpha x) d x, \quad V^{+}(\alpha)=\int_{l}^{\infty} v(x) \exp \{i \alpha(x-l)\} d x, \\
& F(\alpha)=\int_{0}^{l} f(x) \exp (i \alpha x) d x .
\end{aligned}
$$

It can be shown that $U^{-}(\alpha)$ is analytic in the half plane $\tau<\chi(\varepsilon), V^{+}(\alpha)$ is analytic in the half plane $\tau>-\chi(\varepsilon)$ and $F(\alpha)$ is an integral function of $\alpha$. The use of the edge conditions (3.5) ensures that

$$
\begin{aligned}
& U^{-}(\alpha)=O\left(|\alpha|^{-1}\right) \quad \text { as } \quad|\alpha| \rightarrow \infty \quad \text { in } \quad \tau<\chi(\varepsilon), \\
& V^{+}(\alpha)=O\left(|\alpha|^{-1}\right) \text { as } \quad|\alpha| \rightarrow \infty \text { in } \tau>-\chi(\varepsilon), \\
& F(\alpha)=O\left(|\alpha|^{-1}\right) \quad \text { as } \quad|\alpha| \rightarrow \infty \text { in }-\chi(\varepsilon)<\tau<\chi(\varepsilon) .
\end{aligned}
$$

Using Eq.(3.7) in Eqs (3.8) and (3.9) and eliminating $D(\alpha)$, we obtain the following three-part Wiener-Hopf functional relation, for the determination of the three unknown functions $F(\alpha), U^{-}(\alpha)$ and $V^{+}(\alpha)$, as given by

$$
\frac{F(\alpha)}{K(\alpha)}=-\left[A \frac{\exp \{i(\alpha+k) l\}-1}{i(\alpha+k)}+U^{-}(\alpha)+\exp (i \alpha l) V^{+}(\alpha)\right],
$$

valid in the strip $\tau_{-}<\tau<\tau_{+}$, where $\tau_{ \pm}$are chosen such that $-\chi(\varepsilon)<\tau_{-}<0<\tau_{+}<\chi(\varepsilon)$ and

$$
K(\alpha)=\frac{-\frac{g \alpha^{2}}{\omega^{2}}+\frac{i \gamma}{a}+\beta}{\alpha^{6}+\frac{c \alpha^{2}}{\lambda}-\frac{\rho_{0} \omega^{2}}{\lambda}\left(\frac{i \gamma}{a}+\beta\right)} .
$$

To solve the Wiener - Hopf problem described by Eq.(3.12), it is necessary to factorize the function $K(\alpha)$ as $K(\alpha)=K^{+}(\alpha) K^{-}(\alpha)$ where $K^{+}(\alpha)$ is regular in the half plane $\tau>\tau_{-}$and $K^{-}(\alpha)$ is regular in 
the half plane $\tau<\tau_{+}$. For these purpose, the cases $a^{2}<1$ and $a^{2}>1$ are to be considered separately. We note that for $a^{2}<1$ i.e., $\omega<\frac{\omega_{0}}{\sqrt{2}} \equiv \omega_{s}$ and for $a^{2}>1$ i.e., $\omega_{s}<\omega<\omega_{p}$ where

$$
\omega_{p}^{2}=\omega_{s}^{2}\left(1-\frac{\sigma_{1} \omega_{0}^{2}}{2 g \rho_{0}}\right)^{-1}
$$

The denominator of $K(\alpha)$ may be written as (cf. Varlamov (1985))

$$
(\gamma-i a \beta)\left(\gamma-\gamma_{0}\right) \stackrel{4}{\prod_{m=1}}\left(\gamma-\gamma_{m}\right)
$$

The terms $(\lambda-i a \beta)$ do not have zeros for the choice of that branch of $\gamma(\alpha)$ for which $\gamma(0)=-i a \beta$. Also, the factor $\gamma-\gamma_{0}$ does not have zeros when $0<\omega<\omega_{s}$ and has real zeros, say $\pm \alpha_{0}$ when $\omega_{p}<\omega<\omega_{0}$. In the $\alpha$-plane $\gamma_{m}$ represents complex numbers, which are arranged in two symmetric pairs with respect to the imaginary axis. The functions $\gamma-\gamma_{1}$ and $\gamma-\gamma_{2}$ have complex zeros $\pm \alpha_{1}$ and $\pm \alpha_{2}$ respectively, while $\gamma-\gamma_{3}$ and $\gamma-\gamma_{4}$ do not have any zeros. Thus we can write $K(\alpha)$ in Eq.(3.13) as

$$
K(\alpha)=\frac{\frac{g}{\omega^{2}}\left(\gamma+i a \beta \frac{a^{2}-1}{a^{2}+1}\right)}{\left(\gamma-\gamma_{0}\right)\left(\gamma-\gamma_{1}\right)\left(\gamma-\gamma_{2}\right)\left(\gamma-\gamma_{3}\right)\left(\gamma-\gamma_{4}\right)}
$$

$K(\alpha)$ is now factorized for the following two cases.

(a) Case-I: $0<a^{2}<1\left(0<\omega<\omega_{s}\right)$

In this case, we write $K(\alpha)$ as

$$
K(\alpha)=\frac{\frac{g}{\omega^{2}} L(\alpha)\left(\gamma+\gamma_{1}\right)\left(\gamma+\gamma_{2}\right)}{\left(\gamma-\gamma_{0}\right)\left(\alpha^{2}-\alpha_{1}^{2}\right)\left(\alpha^{2}-\alpha_{2}^{2}\right)\left(\gamma-\gamma_{3}\right)\left(\gamma-\gamma_{4}\right)}=\frac{\frac{g}{\omega^{2}} L(\alpha)}{\left(\alpha^{2}-\alpha_{1}^{2}\right)\left(\alpha^{2}-\alpha_{2}^{2}\right) N(\alpha)}
$$

where

and

$$
L(\alpha)=\gamma(\alpha)-i a \beta \frac{1-a^{2}}{1+a^{2}}
$$

$$
N(\alpha)=\frac{\left(\gamma-\gamma_{0}\right)\left(\gamma-\gamma_{3}\right)\left(\gamma-\gamma_{4}\right)}{\left(\gamma+\gamma_{1}\right)\left(\gamma+\gamma_{2}\right)}
$$

Thus

$$
K(\alpha)=K^{+}(\alpha) K^{-}(\alpha), \quad\left(K^{-}(\alpha)=K^{+}(-\alpha)\right)
$$

where

$$
K^{+}(\alpha)=\frac{\left(\frac{g}{\omega^{2}}\right)^{1 / 2} L^{+}(\alpha)}{\left(\alpha+\alpha_{1}\right)\left(\alpha+\alpha_{2}\right) N^{+}(\alpha)}
$$

and 


$$
N(\alpha)=N^{+}(\alpha) N^{-}(\alpha), \quad\left(N^{-}(\alpha)=N^{+}(-\alpha)\right)
$$

The expressions for $N^{+}(\alpha)$ are not written here but it can be shown that

$$
N^{+}(\alpha)=O\left(|\alpha|^{1 / 2}\right) \quad \text { as } \quad|\alpha| \rightarrow \infty \quad \text { for } \quad \tau>\tau_{-}
$$

Thus

$$
\left|K^{+}(\alpha)\right|=O\left(|\alpha|^{-2}\right) \quad \text { as } \quad|\alpha| \rightarrow \infty \quad \text { for } \quad \tau>\tau_{-} .
$$

Now multiplying both sides of Eq.(3.12) by $\frac{\exp (-i \alpha l)}{K^{-}(\alpha)}$ and rearranging, we obtain

$$
A \frac{\exp (i k l) K^{+}(\alpha)}{i(\alpha+k)}-\eta^{+}(\alpha)+\varsigma^{+}(\alpha)+V^{+}(\alpha) K^{+}(\alpha)=-\frac{\exp (-i \alpha l) F(\alpha)}{K^{-}(\alpha)}+\eta^{-}(\alpha)-\varsigma^{-}(\alpha)
$$

where

$$
\varsigma^{+}(\alpha)+\varsigma^{-}(\alpha)=\exp (-i \alpha l) U^{-}(\alpha) K^{+}(\alpha), \quad \eta^{+}(\alpha)+\eta^{-}(\alpha)=A \frac{\exp (-i \alpha l)}{i(\alpha+k)} K^{+}(\alpha)
$$

In Eqs (3.18), $\varsigma^{+}(\alpha), \eta^{+}(\alpha)$ are analytic in $\tau>\tau_{-}$and $\varsigma^{-}(\alpha), \eta^{-}(\alpha)$ are analytic in $\tau<\tau_{+}$and their explicit forms can be obtained by employing the additive decomposition theorem (cf. Noble (1958), pp.13). Similarly, multiplying both sides of Eq.(3.17) by $\frac{1}{K^{+}(\alpha)}$ and rearranging we obtain

$$
\begin{aligned}
& \frac{F(\alpha)}{K^{+}(\alpha)}+R^{+}(\alpha)+S^{+}(\alpha)-\frac{A}{i(\alpha+k)} K^{-}(-k)=-U^{-}(\alpha) K^{-}(\alpha)-R^{-}(\alpha)-S^{-}(\alpha)+ \\
& +\frac{A}{i(\alpha+k)}\left[K^{-}(\alpha)-K^{-}(-k)\right]
\end{aligned}
$$

where

$$
S^{+}(\alpha)+S^{-}(\alpha)=A \frac{\exp \{i(\alpha+k) l\}}{i(\alpha+k)} K^{-}(\alpha), \quad R^{+}(\alpha)+R^{-}(\alpha)=\exp (i \alpha l) V^{+}(\alpha) K^{-}(\alpha)
$$

$R^{+}(\alpha), S^{+}(\alpha)$ being analytic in $\tau>\tau_{-}$and $R^{-}(\alpha), S^{-}(\alpha)$ in $\tau<\tau_{+}$and their explicit forms being obtained by employing the additive decomposition theorem mentioned above.

The left side of Eqs (3.17) and (3.19) are analytic in $\tau>\tau_{-}$while the other sides are analytic in $\tau<\tau_{+}$. Using Eq.(3.11), it is seen that each side of Eqs (3.17) and (3.19) tends to zero as $|\alpha| \rightarrow \infty$ in the appropriate half planes having a common region $\tau_{-}<\tau<\tau_{+}$, so that by Liouville's theorem, each side is identically zero. We are interested in the left hand side of Eq.(3.17) and right hand side of Eq.(3.19).

For brevity, we introduce the notations

$$
\Psi_{*}^{-}=U^{-}(\alpha)-\frac{A}{i(\alpha+k)}, \quad \Psi^{+}=V^{+}(\alpha)+A \frac{\exp (i k l)}{i(\alpha+k)}
$$


where the subscript star is used to indicate that $\Psi_{*}^{-}(\alpha)$ has a pole at $\alpha=-k$, but apart from this, it is analytic in $\tau<\tau_{+}$, and $\Psi^{+}(\alpha)$ is analytic in $\tau>\tau_{-}$. On equating to zero the left side of Eq.(3.17) and the right side of Eq.(3.19) and introducing the explicit expressions for $\varsigma^{+}(\alpha), \eta^{+}(\alpha), R^{-}(\alpha), \varsigma^{-}(\alpha)$ and using the notations (3.21), we obtain

$$
K^{+}(\alpha) \Psi^{+}(\alpha)+\frac{1}{2 \pi i} \int_{-\infty+i c_{l}}^{\infty+i c_{l}} \frac{\exp (-i \xi l) K^{+}(\xi)}{\xi-\alpha} \Psi_{*}^{-}(\xi) d \xi=0, \quad \tau>\tau_{-},
$$

and

$$
K^{-}(\alpha) \Psi_{*}^{-}(\alpha)+\frac{A K^{-}(-k)}{i(\alpha+k)}-\frac{1}{2 \pi i} \int_{-\infty+i d_{l}}^{\infty+i d_{l}} \frac{\exp (i \xi l) K^{-}(\xi)}{\xi-\alpha} \Psi^{+}(\xi) d \xi=0, \quad \tau<\tau_{+}
$$

where $\tau_{-}<c_{1}<0<d_{1}<\tau_{+}$. We choose $c_{l}=-h, d_{l}=h$ where $h$ is positive, then replace $\xi$ by $-\xi$ in Eqs (3.22) and $\alpha$ by $-\alpha$ in Eqs (3.23). Noting that $K^{+}(-\alpha)=K^{-}(\alpha)$, this produces

$$
K^{+}(\alpha) \Psi^{+}(\alpha)-\frac{1}{2 \pi i} \int_{-\infty+i h}^{\infty+i h} \frac{\exp (i \xi l) K^{-}(\xi)}{\xi+\alpha} \Psi_{*}^{-}(-\xi) d \xi=0
$$

and

$$
K^{+}(\alpha) \Psi_{*}^{-}(\alpha)-\frac{A K^{-}(-k)}{i(\alpha-k)}-\frac{1}{2 \pi i} \int_{-\infty+i h}^{\infty+i h} \frac{\exp (i \xi l) K^{-}(\xi)}{\xi+\alpha} \Psi^{+}(\xi) d \xi=0
$$

where now $\tau>-h$ in both the Eqs (3.24) and (3.25). We define

$$
S_{*}^{+}(\alpha)=\Psi^{+}(\alpha)+\Psi_{*}^{-}(-\alpha), \quad D_{*}^{+}(\alpha)=\Psi^{+}(\alpha)-\Psi_{*}^{-}(-\alpha)
$$

where in this case the star denotes that the expressions are analytic in $\tau>\tau_{-}$except for simple pole at $\alpha=k$. Then addition and subtraction of Eqs (3.24) and (3.25) produce

$$
K^{+}(\alpha) S_{*}^{+}(\alpha)-\frac{A K^{+}(k)}{i(\alpha-k)}-\frac{1}{2 \pi i} \int_{-\infty+i h}^{\infty+i h} \frac{\exp (i \xi l) K^{-}(\xi)}{\xi+\alpha} S_{*}^{+}(\xi) d \xi=0, \quad \tau>-h
$$

and

$$
K^{+}(\alpha) D_{*}^{+}(\alpha)+\frac{A K^{+}(k)}{i(\alpha-k)}+\frac{1}{2 \pi i} \int_{-\infty+i h}^{\infty+i h} \frac{\exp (i \xi l) K^{-}(\xi)}{\xi+\alpha} D_{*}^{+}(\xi) d \xi=0, \quad \tau>-h
$$

Equations (3.27) and (3.28) are of the same type and can be treated for approximate solution for large $l$. We write them in a compact form, as given by

$$
K^{+}(\alpha) F_{*}^{l+}(\alpha, \lambda)+\frac{\lambda A K^{+}(k)}{i(\alpha-k)}+\frac{\lambda}{2 \pi i} \int_{-\infty+i h}^{\infty+i h} \frac{\exp (i \xi l) K^{-}(\xi)}{\xi+\alpha} F_{*}^{l+}(\xi, \lambda) d \xi=0, \quad \tau>-h
$$


where $F_{*}^{l+}(\alpha, \lambda)$ is $S_{*}^{+}(\alpha)$ or $D_{*}^{+}(\alpha)$ for $\lambda=-1$ or +1 , so that from Eqs (3.21) and (3.26) to Eqs (3.28) we find that $F_{*}^{l+}(\alpha, \lambda)$ has the form

$$
F_{*}^{l+}(\alpha, \lambda)=F^{l+}(\alpha, \lambda)+\frac{A}{i}\left(\frac{\exp (i k l)}{\alpha+k}-\frac{\lambda}{\alpha-k}\right)
$$

where $F^{1+}(\alpha, \lambda)$ is analytic in $\tau>\tau_{-}$, it being understood that $F^{1+}(\alpha, 1)=V^{+}(\alpha)-U^{-}(-\alpha)$ and $F^{l+}(\alpha,-l)=V^{+}(\alpha)+U^{-}(-\alpha)$.

Now writing, in the integrand of the integral in the left hand side of Eq.(3.29)

$$
K^{-}(\xi)=\frac{1}{K^{+}(\xi)}\left[-\frac{g}{\omega^{2}}+\frac{1}{\xi^{2}-\alpha_{0}^{2}}\left\{A_{l}+B_{l} \gamma(\xi)\right\}\right]
$$

where

$$
A_{1}=-2 a^{2} \beta^{2} \frac{\sigma_{1} \omega^{2}}{\left(1+a^{2}\right) c}\left(1-\frac{2 g \rho_{0}}{\left(1+a^{2}\right) c}\right) \frac{g}{\omega^{2}}, \quad B_{1}=-2 i a \beta \frac{\sigma_{1} g}{\left(1+a^{2}\right) c},
$$

it is clear that the integrand consists of two types of terms, the first type involves simple poles while the other type involves branch points at $\xi= \pm a \beta$ in the complex $\xi$-plane. In the presence of simple poles the integrals are evaluated by using the residue theorem after completing the contour by a semi-circle of a large radius in the upper half plane, and to evaluate the integrals involving the branch points, only one branch point, viz. $\xi=a \beta$ needs to be considered and as such a branch cut is taken parallel to the positive imaginary axis from $\xi=a \beta$ to infinity. Then the contour is deformed into the two sides of the branch cut and contributions from the poles, if any, are taken into account. The contributions from the two sides of the branch cut involve integrals of the form

$$
\int_{0}^{\infty} \psi(u) u^{1 / 2} \exp (-u l) d u
$$

where $\psi(u)$ is an analytic function. If $I(l)$ denotes the integral (3.32) and for large $l$, it becomes asymptotically equal to

$$
I(l) \approx \frac{\pi^{1 / 2}}{2}\left(\frac{1}{l}\right)^{3 / 2} \psi\left(\frac{3}{2 l}\right)+O\left(l^{-7 / 2}\right) .
$$

Incorporating the aforesaid method we find that for large $l$,

$$
\int_{-\infty+i h}^{\infty+i h} \frac{\exp (i \xi l) K^{-}(\xi)}{\xi+\alpha} F^{1+}(\xi, \lambda) d \xi \approx 2 \pi i\left[T_{l}(\alpha) F^{1+}\left(\alpha_{0}, \lambda\right)+T(\alpha) F^{1+}\left(\varepsilon^{\prime}, \lambda\right)\right]
$$

where

$$
T(\alpha)=-\pi^{1 / 2}\left(\frac{l}{l}\right)^{3 / 2} \frac{\exp (i l a \beta) \sqrt{a \beta+\varepsilon^{\prime}} \exp \left\{\frac{3 \pi i}{4}\right\}}{2 \pi i\left(\alpha+\varepsilon^{\prime}\right)\left(\varepsilon^{\prime 2}-\alpha_{0}^{2}\right) K^{+}\left(\varepsilon^{\prime}\right)},
$$


Similarly

$$
T_{l}(\alpha)=\frac{\exp \left(i l \alpha_{0}\right)}{2 \alpha_{0}\left(\alpha_{0}+\alpha\right) K^{+}\left(\alpha_{0}\right)}\left(A_{1}+B_{1} \sqrt{\alpha_{0}^{2}-a^{2} \beta^{2}}\right), \quad \varepsilon^{\prime}=\varepsilon+\frac{3 i}{2 l}
$$

$$
\int_{-\infty+i h}^{\infty+i h} \frac{\exp (i \xi l) K^{-}(\xi)}{(\xi+\alpha)(\xi+k)} d \xi \approx 2 \pi i\left[\frac{T_{l}(\alpha)}{\alpha_{0}+k}+R_{l}(\alpha)\right]
$$

where $R_{l}(\alpha)=\frac{T(\alpha)}{\varepsilon^{\prime}+k}$ and

$$
\int_{-\infty+i h}^{\infty+i h} \frac{\exp (i \xi l) K^{-}(\xi)}{(\xi+\alpha)(\xi-k)} d \xi \approx 2 \pi i\left[\frac{T_{2}(\alpha)}{\alpha_{0}-k}+R_{2}(\alpha)\right]
$$

where $R_{2}(\alpha)=\frac{T(\alpha)}{\varepsilon^{\prime}-k}$ and

$$
\begin{aligned}
& T_{2}(\alpha)=\frac{1}{\alpha+k}\left\{-\frac{\exp (i k l)}{K^{+}(k)}\left(\frac{g}{\omega^{2}}\left(\alpha_{0}-k\right)+\frac{A_{1}}{\alpha_{0}+k}+\frac{B_{1} \sqrt{k^{2}-a^{2} \beta^{2}}}{\alpha_{0}+k}\right)\right\}+ \\
& +\frac{1}{\alpha+\alpha_{0}}\left\{\frac{\exp \left(i \alpha_{0} l\right)}{2 \alpha_{0} K^{+}\left(\alpha_{0}\right)}\left(A_{1}+B_{I} \sqrt{\alpha_{0}^{2}-a^{2} \beta^{2}}\right)\right\} .
\end{aligned}
$$

Using results (3.34), (3.35) and (3.36) for large $l$ in Eqs (3.29), we obtain an approximate relation between the function $F^{1+}(\alpha, \lambda)$ and the unknown quantities $F^{1+}\left(\alpha_{0}, \lambda\right), F^{l+}\left(\varepsilon^{\prime}, \lambda\right)$. Setting $\alpha=\alpha_{0}$ and $\alpha=\varepsilon^{\prime}$ in this we get two equations involving these two unknowns, which, when solved, produce

$$
\begin{aligned}
& F^{l+}\left(\alpha_{0}, \lambda\right)=\frac{A}{i\left(A_{\lambda} D_{\lambda}-B_{\lambda} C_{\lambda}\right)}\left[\exp (i k l)\left(S_{\lambda} B_{\lambda}-Q_{\lambda} D_{\lambda}\right)+T_{\lambda} B_{\lambda}-R_{\lambda} D_{\lambda}\right], \\
& A_{\lambda}=K^{+}\left(\alpha_{0}\right)+\lambda T_{1}\left(\alpha_{0}\right), \quad B_{\lambda}=\lambda T\left(\alpha_{0}\right), \\
& F^{l+}\left(\varepsilon^{\prime}, \lambda\right) \approx \frac{A}{i\left(A_{\lambda} D_{\lambda}-B_{\lambda} C_{\lambda}\right)}\left[\exp (i k l)\left(S_{\lambda} A_{\lambda}-Q_{\lambda} C_{\lambda}\right)+T_{\lambda} A_{\lambda}-R_{\lambda} C_{\lambda}\right], \\
& C_{\lambda}=\lambda T_{1}\left(\varepsilon^{\prime}\right), \quad D_{\lambda}=K^{+}\left(\varepsilon^{\prime}\right)+\lambda T\left(\varepsilon^{\prime}\right), \quad Q_{\lambda}=\frac{K^{+}\left(\alpha_{0}\right)}{\alpha_{0}+k}+\lambda\left\{\frac{T_{1}\left(\alpha_{0}\right)}{\alpha_{0}+k}+R_{l}\left(\alpha_{0}\right)\right\}, \\
& R_{\lambda}=\lambda^{2}\left\{\frac{T_{2}\left(\alpha_{0}\right)}{\alpha_{0}-k}+R_{2}\left(\alpha_{0}\right)\right\}+\lambda\left\{\frac{K^{+}(k)}{\alpha_{0}-k}+\frac{K^{+}\left(\alpha_{0}\right)}{\alpha_{0}-k}\right\}, \\
& S_{\lambda}=\frac{K^{+}\left(\varepsilon^{\prime}\right)}{\varepsilon^{\prime}+k}+\lambda\left\{\frac{T_{l}\left(\varepsilon^{\prime}\right)}{\alpha_{0}+k}+R_{l}\left(\varepsilon^{\prime}\right)\right\}, T_{\lambda}=\lambda^{2}\left\{\frac{T_{2}\left(\varepsilon^{\prime}\right)}{\alpha_{0}-k}+R_{2}\left(\varepsilon^{\prime}\right)\right\}+\lambda\left\{\frac{K^{+}(k)}{\varepsilon^{\prime}-k}+\frac{K^{+}\left(\varepsilon^{\prime}\right)}{\varepsilon^{\prime}-k}\right\} .
\end{aligned}
$$


Thus $F^{l+}(\alpha, \lambda)$ is obtained for large $l$ and is given by

$$
\begin{aligned}
& F^{l+}(\alpha, \lambda) \approx \frac{1}{K^{+}(\alpha)}\left[-\frac{\lambda A K^{+}(k)}{i(\alpha-k)}-\frac{\lambda A \exp (i k l)}{i}\left(\frac{T_{1}(\alpha)}{\alpha_{0}+k}+R_{l}(\alpha)\right)-\frac{\lambda^{2} A}{i}\left(\frac{T_{2}(\alpha)}{\alpha_{0}-k}+R_{2}(\alpha)\right)+\right. \\
& \left.-K^{+}(\alpha)\left(\frac{A \exp (i k l)}{i(\alpha+k)}-\frac{\lambda A}{i(\alpha-k)}\right)-\lambda\left\{T_{l}(\alpha) F^{l+}\left(\alpha_{0}, \lambda\right)+T(\alpha) F^{l+}\left(\varepsilon^{\prime}, \lambda\right)\right\}\right]
\end{aligned}
$$

where $F^{l+}\left(\alpha_{0}, \lambda\right)$ and $F^{1+}\left(\varepsilon^{\prime}, \lambda\right)$ are given in Eqs (3.37) and (3.38), respectively. Putting $\lambda=-1$ and 1 in Eq.(3.40) we obtain two equations for $V^{+}(\alpha)+U^{-}(\alpha)$ and $V^{+}(\alpha)-U^{-}(\alpha)$. By addition and subtraction we find $V^{+}(\alpha)$ and $U^{-}(\alpha)$ for large $l$. Replacing $\alpha$ by $-\alpha$ we obtain $U^{\alpha}$. Thus $V^{+}(\alpha)$ and $U^{+}(\alpha)$ are obtained for large $l$. Now the use of Eqs (3.7) in Eq.(3.8) produces $D(\alpha)$. Thus we obtain $D(\alpha)$ as

$$
\begin{aligned}
& D(\alpha)=\frac{H K^{+}(k)}{(\alpha+k)(\gamma-i a \beta) N^{+}(\alpha) L^{-}(\alpha)}+\frac{\left(\frac{\omega^{2}}{g}\right)^{1 / 2}}{i(\gamma-i a \beta)}\left[\frac { 1 } { L ^ { - } ( \alpha ) N ^ { + } ( \alpha ) } \left\{\frac{A}{i}\left(\exp (i k l) R_{l}(-\alpha)+\frac{T_{1}(-\alpha)}{\alpha_{0}+k}\right)+\right.\right. \\
& \left.\left.+C_{3} T(-\alpha)+C_{4} T_{1}(-\alpha)\right\}-\frac{\exp (i \alpha l)}{N^{-}(\alpha) L^{+}(\alpha)}\left\{\frac{A}{i}\left(R_{2}(\alpha)+\frac{T_{2}(\alpha)}{\alpha_{0}-k}\right)+C_{2} T_{l}(\alpha)+C_{l} T(\alpha)\right\}\right], \quad 0<\omega \leq \omega_{s}
\end{aligned}
$$

where

$$
\begin{aligned}
& C_{1}=F^{1+}\left(\varepsilon^{\prime}, 1\right)-F^{l+}\left(\varepsilon^{\prime},-1\right), \quad C_{2}=F^{l+}\left(\alpha_{0}, l\right)-F^{l+}\left(\alpha_{0},-1\right), \\
& C_{3}=F^{l+}\left(\varepsilon^{\prime}, 1\right)+F^{l+}\left(\varepsilon^{\prime},-1\right), \quad C_{4}=F^{l+}\left(\alpha_{0}, l\right)+F^{l+}\left(\alpha_{0}, l\right), \quad H=i\left(\frac{\omega^{2}}{g}\right)^{1 / 2} A .
\end{aligned}
$$

(b) Case-II: $a^{2}>1,\left(\omega_{s}<\omega<\omega_{0}\right)$

We find that in this case two sub-cases arising from $\omega<\omega_{p}$ and $\omega>\omega_{p}$ where $\omega_{s}<\omega_{p}<\omega_{0}$. (i) $\omega_{s}<\omega \leq \omega_{p}$

In this sub-case the numerator of $K(\alpha)$ has zeros at $\alpha= \pm \alpha_{s}$ for our choice of the branch $\gamma(\varepsilon)$. Thus we write

$$
\begin{aligned}
& K(\alpha)=\frac{\frac{g}{\omega^{2}}\left(\alpha^{2}-\alpha_{s}^{2}\right)\left(\gamma+\gamma_{1}\right)\left(\gamma+\gamma_{2}\right)}{\left(\gamma-\gamma_{0}\right)\left(\alpha^{2}-\alpha_{1}^{2}\right)\left(\alpha^{2}-\alpha_{2}^{2}\right)\left(\gamma-\gamma_{3}\right)\left(\gamma-\gamma_{4}\right) M(\alpha)}= \\
& =\frac{\frac{g}{\omega^{2}}\left(\alpha^{2}-\alpha_{s}^{2}\right)}{\left(\alpha^{2}-\alpha_{1}^{2}\right)\left(\alpha^{2}-\alpha_{2}^{2}\right) M(\alpha) N(\alpha)}
\end{aligned}
$$

where

$$
M(\alpha)=\gamma(\alpha)-i a \beta \frac{a^{2}-1}{a^{2}+1}
$$

Thus 


$$
K^{+}(\alpha)=\frac{\left(\frac{g}{\omega^{2}}\right)^{1 / 2}\left(\alpha+\alpha_{s}\right)}{\left(\alpha+\alpha_{1}\right)\left(\alpha+\alpha_{2}\right) M^{+}(\alpha) N^{+}(\alpha)}
$$

Proceeding as before, $D(\alpha)$ in this case is obtained as

$D(\alpha)=\frac{H K^{+}(k) M^{-}(\alpha)}{(\alpha+k)(\gamma-i a \beta)\left(\alpha-\alpha_{s}\right) N^{+}(\alpha)}+\frac{\left(\frac{\omega^{2}}{g}\right)^{1 / 2} M^{-}(\alpha)}{i(\gamma-i a \beta)\left(\alpha-\alpha_{s}\right) N^{+}(\alpha)}\left\{\frac{A}{i}\left(\exp (i k l) R_{l}(-\alpha)+\frac{T_{l}(-\alpha)}{\alpha_{0}+k}\right)+\right.$

$\left.+C_{3} T(-\alpha)+C_{4} T_{l}(-\alpha)\right\}-\frac{\left(\frac{\omega^{2}}{g}\right)^{1 / 2} \exp (i \alpha l) M^{+}(\alpha)}{i(\gamma-i a \beta)\left(\alpha+\alpha_{s}\right) N^{-}(\alpha)}\left\{\frac{A}{i}\left(R_{2}(\alpha)+\frac{T_{2}(\alpha)}{\alpha_{0}-k}\right)+C_{2} T_{l}(\alpha)+C_{l} T(\alpha)\right\}, \quad \omega_{s}<\omega \leq \omega_{p}$.

(ii) $\omega_{p}<\omega<\omega_{0}$

In this case, we can write

$$
\begin{aligned}
& K(\alpha)=\frac{\frac{g}{\omega^{2}}\left(\alpha^{2}-\alpha_{s}^{2}\right)\left(\gamma+\gamma_{0}\right)\left(\gamma+\gamma_{1}\right)\left(\gamma+\gamma_{2}\right)}{\left(\alpha^{2}-\alpha_{0}^{2}\right)\left(\alpha^{2}-\alpha_{1}^{2}\right)\left(\alpha^{2}-\alpha_{2}^{2}\right)\left(\gamma-\gamma_{3}\right)\left(\gamma-\gamma_{4}\right) M(\alpha)}= \\
& =\frac{\frac{g}{\omega^{2}}\left(\alpha^{2}-\alpha_{s}^{2}\right) P(\alpha)}{\left(\alpha^{2}-\alpha_{0}^{2}\right)\left(\alpha^{2}-\alpha_{1}^{2}\right)\left(\alpha^{2}-\alpha_{2}^{2}\right) M(\alpha)}
\end{aligned}
$$

where

$$
P(\alpha)=\frac{\left(\gamma+\gamma_{0}\right)\left(\gamma+\gamma_{1}\right)\left(\gamma+\gamma_{2}\right)}{\left(\gamma-\gamma_{3}\right)\left(\gamma-\gamma_{4}\right)}
$$

Thus

$$
K^{+}(\alpha)=\frac{\left(\frac{g}{\omega^{2}}\right)^{1 / 2}\left(\alpha+\alpha_{s}\right) P^{+}(\alpha)}{\left(\alpha+\alpha_{0}\right)\left(\alpha+\alpha_{1}\right)\left(\alpha+\alpha_{2}\right) M^{+}(\alpha)}
$$

Finally, $D(\alpha)$ in this case is obtained as

$$
\begin{aligned}
& D(\alpha)=\frac{H K^{+}(k) P^{+}(\alpha) M^{-}(\alpha)}{(\alpha+k)(\gamma-i a \beta)\left(\alpha-\alpha_{s}\right)\left(\alpha+\alpha_{0}\right)}+\frac{\left(\frac{\omega^{2}}{g}\right)^{1 / 2} P^{+}(\alpha) M^{-}(\alpha)}{i(\gamma-i a \beta)\left(\alpha-\alpha_{s}\right)\left(\alpha+\alpha_{0}\right)}\left\{\frac{A}{i}\left(\exp (i k l) R_{l}(-\alpha)+\frac{T_{l}(-\alpha)}{\alpha_{0}+k}\right)+\right. \\
& \left.+C_{3} T(-\alpha)+C_{4} T_{l}(-\alpha)\right\}-\frac{\left(\frac{\omega^{2}}{g}\right)^{1 / 2} P^{-}(\alpha) M^{-}(\alpha)}{i(\gamma-i a \beta)\left(\alpha+\alpha_{s}\right)\left(\alpha-\alpha_{0}\right)}\left\{\frac{A}{i}\left(R_{2}(\alpha)+\frac{T_{2}(\alpha)}{\alpha_{0}-k}\right)+C_{2} T_{l}(\alpha)+C_{l} T(\alpha)\right\}, \omega_{p}<\omega<\omega_{0} .
\end{aligned}
$$


Now rewriting Eqs (3.41), (3.46) and (3.47) in a compact form and using Eq.(3.7) we obtain $\phi(x, y)$ for large $l$ after taking the Fourier inversion, as given by

$$
\begin{aligned}
& \phi(x, y)=\frac{H K^{+}(k)}{2 \pi} \int_{-\infty}^{\infty} \frac{\Omega_{l}(\alpha) \exp \left(\frac{i \gamma(\alpha) y}{a}-i \alpha x\right)}{(\alpha+k)(\gamma-i a \beta) \Omega_{2}(\alpha)} d \alpha+\frac{\left(\frac{\omega^{2}}{g}\right)^{1 / 2}}{2 \pi i} \int_{-\infty}^{\infty} \frac{\Omega_{l}(\alpha) Q(\alpha) \exp \left(\frac{i \gamma(\alpha) y}{a}-i \alpha x\right)}{(\gamma-i a \beta) \Omega_{2}(\alpha)} d \alpha+ \\
& -\frac{\left(\frac{\omega^{2}}{g}\right)^{1 / 2}}{2 \pi i} \int_{-\infty}^{\infty} \frac{\Omega_{l}(-\alpha) R(\alpha) \exp \left(\frac{i \gamma(\alpha) y}{a}-i \alpha(x-l)\right)}{(\gamma-i a \beta) \Omega_{3}(\alpha)} d \alpha
\end{aligned}
$$

where

$$
\left.\begin{array}{l}
\Omega_{l}(\alpha) \\
\Omega_{2}(\alpha) \\
\Omega_{3}(\alpha)
\end{array}\right\}=\left\{\begin{array}{l}
\left\{\begin{array}{l}
1 \\
L^{-}(\alpha) N^{+}(\alpha)\left(\alpha+\alpha_{1}\right)\left(\alpha+\alpha_{2}\right) ; \quad 0<\omega \leq \omega_{s} \\
L^{+}(\alpha) N^{-}(\alpha)\left(\alpha-\alpha_{1}\right)\left(\alpha-\alpha_{2}\right)
\end{array}\right. \\
\left\{\begin{array}{l}
M^{-}(\alpha) \\
\left(\alpha-\alpha_{s}\right)\left(\alpha+\alpha_{1}\right)\left(\alpha-\alpha_{2}\right) N^{+}(\alpha) ; \quad \omega_{s}<\omega \leq \omega_{p}
\end{array}\right. \\
\left(\alpha+\alpha_{s}\right)\left(\alpha-\alpha_{1}\right)\left(\alpha-\alpha_{2}\right) N^{-}(\alpha) \\
\left\{\begin{array}{l}
P^{+}(\alpha) M^{-}(\alpha) \\
\left(\alpha+\alpha_{0}\right)\left(\alpha-\alpha_{s}\right)\left(\alpha+\alpha_{1}\right)\left(\alpha+\alpha_{2}\right) ; \quad \omega_{p}<\omega<\omega_{0} \\
\left(\alpha-\alpha_{0}\right)\left(\alpha+\alpha_{s}\right)\left(\alpha-\alpha_{1}\right)\left(\alpha-\alpha_{2}\right)
\end{array}\right.
\end{array}\right.
$$

and

$$
\begin{aligned}
& Q(\alpha)=\frac{A}{i}\left(\exp (i k l) R_{l}(-\alpha)+\frac{T_{l}(-\alpha)}{\alpha_{0}+k}\right)+C_{3} T(-\alpha)+C_{4} T_{l}(-\alpha), \\
& R(\alpha)=\frac{A}{i}\left(R_{2}(\alpha)+\frac{T_{2}(\alpha)}{\alpha_{0}-k}\right)+C_{2} T_{l}(\alpha)+C_{l} T(\alpha),
\end{aligned}
$$

and $K^{+}(k)$ having appropriate values in different ranges of $\omega$ after making $\varepsilon \rightarrow 0$. The integration in Eq.(3.48) is taken along the real axis of the $\alpha$-plane with indentation above the negative poles and below the positive poles. In the next section we analyze the integrals in Eq.(3.48) asymptotically, for a large distance from the edges of the strip.

\section{Asymptotic analysis of the solutions}

For an asymptotic analysis of the integrals in Eq.(3.48), we introduce the polar co-ordinates $\left(r_{-}, \theta_{-}\right)$ and $\left(r_{+}, \theta_{+}\right)\left(0<\theta_{ \pm}<\pi\right)$, with centers at the end points $(0,0)$ and $(l, 0)$ of the strip, respectively. For 
simplicity, we write the polar co-ordinate in the form $\left(r_{n}, \theta_{n}\right)$ where $n= \pm, 0<\theta_{n}<\pi$ and the angles $\theta_{n}$ are measured in a clockwise sense from the $x$-axis. We note that the characteristic equation of the PDE (2.6) represents a pair of straight lines $y= \pm a x$ and $y= \pm a(x-l)$ and forms a characteristic cone at the two ends of the strip, respectively (cf. Fig.1). These straight lines are inclined at angles $\theta_{c}$ and $\pi-\theta_{c}$ at points $(0,0)$ and $(0, l)$ with the $x$-axis, respectively, where $\theta_{c}$ is defined by $\tan \theta_{c}=a$. Let $\theta_{0}$ be defined by $\tan \theta_{0}=\frac{a^{2} b}{k}$, and then $\theta_{0}$ is the angle which the group velocity vector of the incident wave field $\phi_{0}$ makes with the $x$-axis.

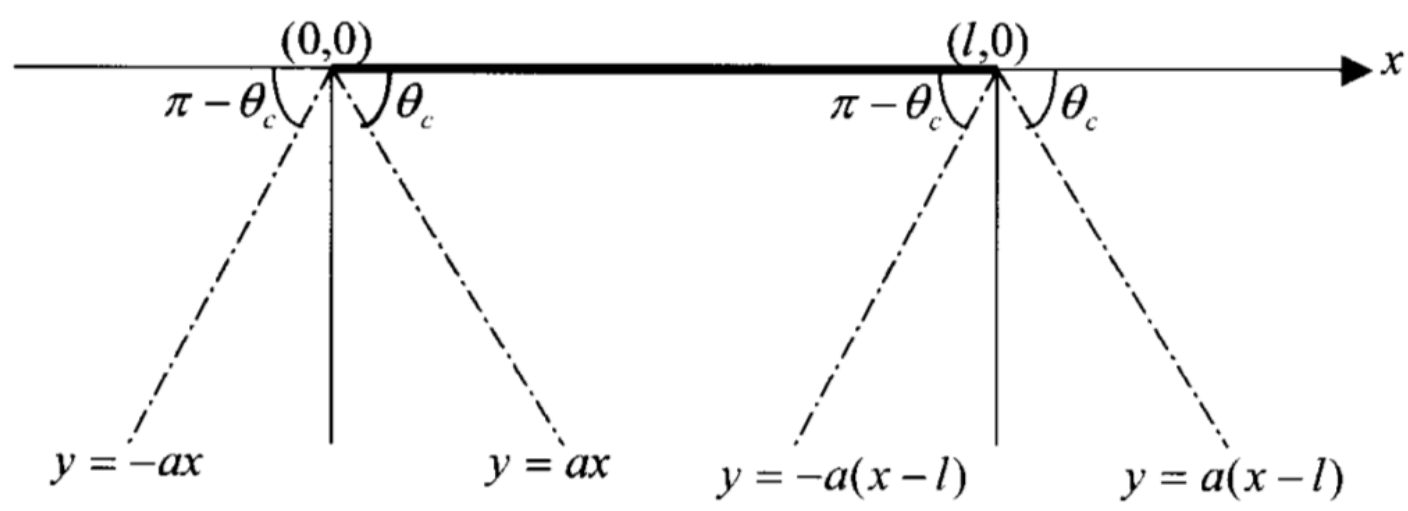

Fig.1. Representation of characteristic equations at the ends of the elastic strip.

From the representation (3.48) it follows that $\phi(x, y)$ is continuous in the region $y \leq 0$ together with its derivatives upto the third order. The fourth order derivative of this function has a logarithmic singularity on the characteristics $\theta=\theta_{c}$ and $\theta=\pi-\theta_{c}$ which passes through the two ends of the strip $(0,0)$ and $(l, 0)$. The fifth and sixth order derivatives of the solution have a singularity of the order $\left|r \sin \left(\theta-\theta_{c}\right)\right|^{-1}$ and $\left|r \cos \left(\theta-\theta_{c}\right)\right|^{-1}$ respectively on the above mentioned characteristics.

Now it is convenient to write the integral in terms of polar co-ordinates $r_{n}$ and $\theta_{n}$, then

$$
\varphi\left(r_{n}, \theta_{n}\right)=\int_{-\infty}^{\infty} \chi_{l}(\alpha) \exp \left(-i r_{n} f(\alpha)\right) d \alpha
$$

where

$$
f(\alpha)=\alpha \cos \theta_{n}+\frac{\sqrt{\alpha^{2}-a^{2} \beta^{2}}}{a} \sin \theta_{n},
$$

and

$$
\chi_{l}(\alpha)=\frac{H K^{+}(k)}{2 \pi} \frac{\Omega_{l}(\alpha)}{(\alpha+k)(\gamma-i a \beta) \Omega_{2}(\alpha)}+\frac{\left(\frac{\omega^{2}}{g}\right)^{1 / 2}}{2 \pi i}\left\{\frac{\Omega_{l}(\alpha) Q(\alpha)}{(\gamma-i a \beta) \Omega_{2}(\alpha)}-\frac{\Omega_{l}(-\alpha) R(\alpha)}{(\gamma-i a \beta) \Omega_{3}(\alpha)}\right\} .
$$

Saddle points of $\operatorname{Re} f(\alpha)$ occur when $f^{\prime}(\alpha)=0$, that is, when 


$$
\frac{\alpha}{a \sqrt{\alpha^{2}-a^{2} \beta^{2}}} \sin \theta_{n}=-\cos \theta_{n}
$$

The solutions of this equation corresponding to the principal value of $\sqrt{\alpha^{2}-a^{2} \beta^{2}}$ are

$$
\begin{aligned}
& \alpha_{n}^{1}=-\frac{a^{2} \beta \cos \theta_{n}}{\Phi\left(\theta_{n}\right)} \text { where } \Phi\left(\theta_{n}\right)=\left(a^{2} \cos ^{2} \theta_{n}-\sin ^{2} \theta_{n}\right)^{1 / 2}>0, \\
& \alpha_{n}^{2}=\frac{i a^{2} \beta \cos \theta_{n}}{\Psi\left(\theta_{n}\right)} \text { where } \Psi\left(\theta_{n}\right)=\left(\sin ^{2} \theta_{n}-a^{2} \cos ^{2} \theta_{n}\right)^{1 / 2}>0 .
\end{aligned}
$$

For the saddle point $\alpha_{n}^{l}$

$$
f\left(\alpha_{n}^{1}\right)=-\beta \Phi\left(\theta_{n}\right) \quad \text { and } \quad f^{\prime \prime}\left(\alpha_{n}^{1}\right)=-\frac{\Phi^{3}\left(\theta_{n}\right)}{a^{2} \beta \sin ^{2} \theta_{n}}>0 .
$$

To apply the method of the steepest descent, we deform the path of integration into a contour $\Gamma$ which passes through the saddle point such that $i\left\{f(\alpha)-f\left(\alpha_{n}^{l}\right)\right\}$ is real and negative or zero on $\Gamma$. We define a continuous real variable $t_{l}$ by the equation

$$
-i\left\{f(\alpha)-f\left(\alpha_{n}^{1}\right)\right\}=-\frac{1}{2} t_{l}^{2}
$$

for values of $\alpha$ on $\Gamma$.

To determine the sign of $t_{1}$, we assume $t_{1}$ to increase steadily as $\alpha$ moves along the path and it is clear that $t_{1}$ vanishes only when $\alpha=\alpha_{n}^{l}$. Then by the change of variable $\alpha$ to $t_{1}$, the integral taken along $\Gamma$ becomes

$$
\varphi_{\Gamma}\left(r_{n}, \theta_{n}\right)=\exp \left\{-i r_{n} f\left(\alpha_{n}^{l}\right)\right\} \int_{-\infty}^{\infty} \exp \left\{-\frac{1}{2} r_{n} t_{l}^{2}\right\} \chi_{l}(\alpha) \frac{d \alpha}{d t_{1}} d t_{l}
$$

Near the saddle point $f^{\prime}\left(\alpha_{n}^{1}\right)=0$, we get approximately

$$
-i\left\{f(\alpha)-f\left(\alpha_{n}^{l}\right)\right\} \approx-i\left[\frac{1}{2}\left(\alpha-\alpha_{n}\right)^{2} f^{\prime \prime}\left(\alpha_{n}^{l}\right)\right] \text {, }
$$

so that we obtain from Eqs (4.2), (4.3), (4.5)

$$
\alpha-\alpha_{n}^{1} \approx \pm \frac{a t_{1} \sin \theta_{n}}{\Phi\left(\theta_{n}\right)} \sqrt{\frac{\beta}{\Phi\left(\theta_{n}\right)}} \exp \left(\frac{i \pi}{4}\right) .
$$

The sign is to be determined with reference to the sense in which the curve passes through the saddle point, and we are thus led to consider the general form of the deformed contour.

Points at which the path crosses the real $\alpha$-axis are given by the equation

$$
\operatorname{Im}\left\{f(\alpha)-f\left(\alpha_{n}^{1}\right)\right\}=0 \quad(\alpha \text { real }),
$$


and it is found that, apart from the saddle point, there is only one such point, given by

$$
\alpha_{n}^{l}=-\frac{\beta\left(a^{2} \cos ^{2} \theta_{n}-\sin ^{2} \theta_{n}\right)}{\cos \theta_{n}}
$$

The asymptotic behaviour of the two branches of the path is represented by the equations

$$
\operatorname{Im}\left[\alpha\left\{-i\left( \pm \frac{\sin \theta_{n}}{a}\right)-i \cos \theta_{n}\right\}\right]=\beta \Phi\left(\theta_{n}\right) .
$$

If $0<\theta_{n}<\pi / 2$, the path has the two asymptotes

$$
\sigma=-\beta a\left(\frac{a \cos \theta_{n}-\sin \theta_{n}}{a \cos \theta_{n}+\sin \theta_{n}}\right)=\sigma_{l} \text { (say) and } \sigma=-\beta a\left(\frac{a \cos \theta_{n}+\sin \theta_{n}}{a \cos \theta_{n}-\sin \theta_{n}}\right)=\sigma_{2} \text { (say), } \sigma_{2}<\sigma_{1} \text {. }
$$

The general form of the contour $\Gamma$ when $0<\theta_{-}<\pi / 2$ and passing through the saddle point $\alpha_{-}^{l}$, is shown in Fig.2.

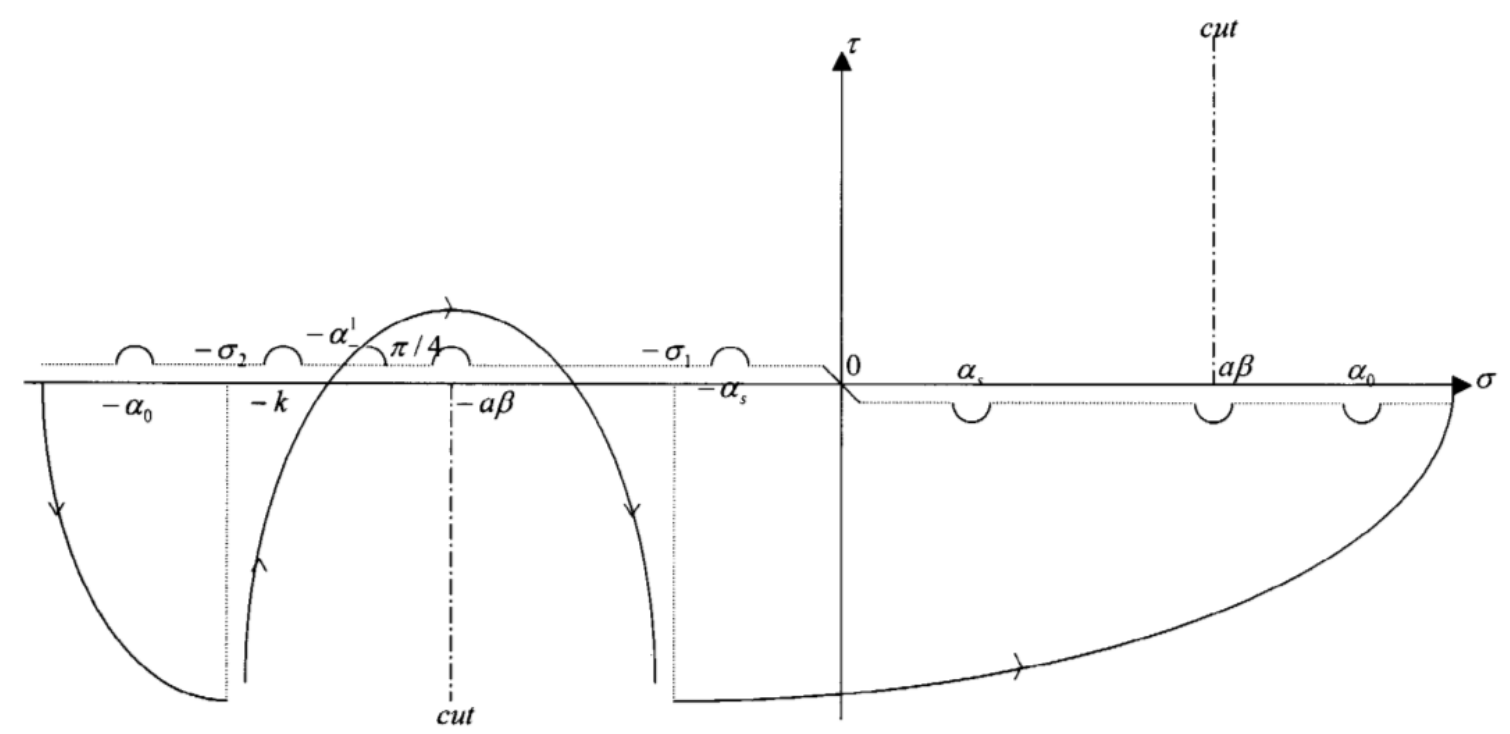

Fig.2. Representation of contour passing through the saddle point in $(0, \pi / 2)$.

It is clear that the positive sign must be taken in formula (4.6) and if we substitute this expression for $\alpha-\alpha_{-}^{1}$ in integral (4.4) we readily obtain the dominant term of its asymptotic expansion. When $0<\theta_{+}<\pi / 2$, i.e., the contour passes through the saddle point $\alpha_{+}^{l}$, in a similar way we can obtain the dominant term of its asymptotic expansion. Thus for $0<\theta_{n}<\pi / 2$, the asymptotic expansion denoted by $\varphi_{n}^{l}$ is obtained as

$$
\phi_{n}^{l} \approx \chi_{1}\left(\alpha_{n}^{l}\right) \frac{a \beta \sin \theta_{n}}{\Phi^{3 / 2}\left(\theta_{n}\right)} \sqrt{\frac{2 \pi}{\beta r_{n}}} \exp \left\{i r_{n} \beta \Phi\left(\theta_{n}\right)+\frac{i \pi}{4}\right\}+O\left(\frac{1}{\beta r_{n}}\right) .
$$


In the integral (3.48), the pole of the integrand occurs on the real axis at the point $\alpha=-k$. For $0<\theta_{-}<\pi / 2$, the deformation of the contour when passing through the saddle point $\alpha=\alpha_{-}^{l}$ is indicated in Fig.2. From this figure, it is seen that the contribution of the pole $\alpha=-k$ is to be considered here if $\theta_{-}<\theta_{0}$. Similarly, if the contour passes through the point $\alpha=\alpha_{+}^{l}$, then the contribution of the above pole is to be considered if $\theta_{+}>\theta_{0}$. Thus we obtain in this case

where

$$
\varphi(x, y)=\varphi_{2}-\varphi_{1} \quad \text { (say) }
$$

$$
\phi_{2}=-\frac{-\lambda k^{6}+c k^{2}+\rho_{0} \omega^{2}(i b-\beta)}{\lambda k^{6}+c k^{2}-\rho_{0} \omega^{2}(i b+\beta)} \exp (i b y+i k x),
$$

represents the wave reflected from the strip and $\varphi_{1}$ is the wave reflected from the free surface.

Again, the deformation of the same contour for $\theta_{n}$ ranging from $\pi / 2$ to $\pi$ is shown in Fig.3 and we determine $\varphi_{n}^{l}$ similarly. Also, from integral (3.48), we have seen that $\omega$ varies from 0 to $\omega_{s}$ i.e., in $0<\theta_{c}<\pi / 4$ and $\pi-\frac{\pi}{4}<\theta_{c}<\pi$. Again, from the saddle points, it may be noted that $\Phi\left(\theta_{n}\right)$, i.e., $\left(a^{2} \cos ^{2} \theta_{n}-\sin ^{2} \theta_{n}\right)^{1 / 2}>0$ i.e., $0<\theta_{n}<\theta_{c}$ and $\pi-\theta_{c}<\theta_{n}<\pi$. Thus we conclude that $\varphi_{n}^{l}$ is the diffraction term arising in the region within the characteristic cone. It represents waves whose amplitude decays away from the edges of the strip like $\left(\beta r_{n}\right)^{-1 / 2}$.

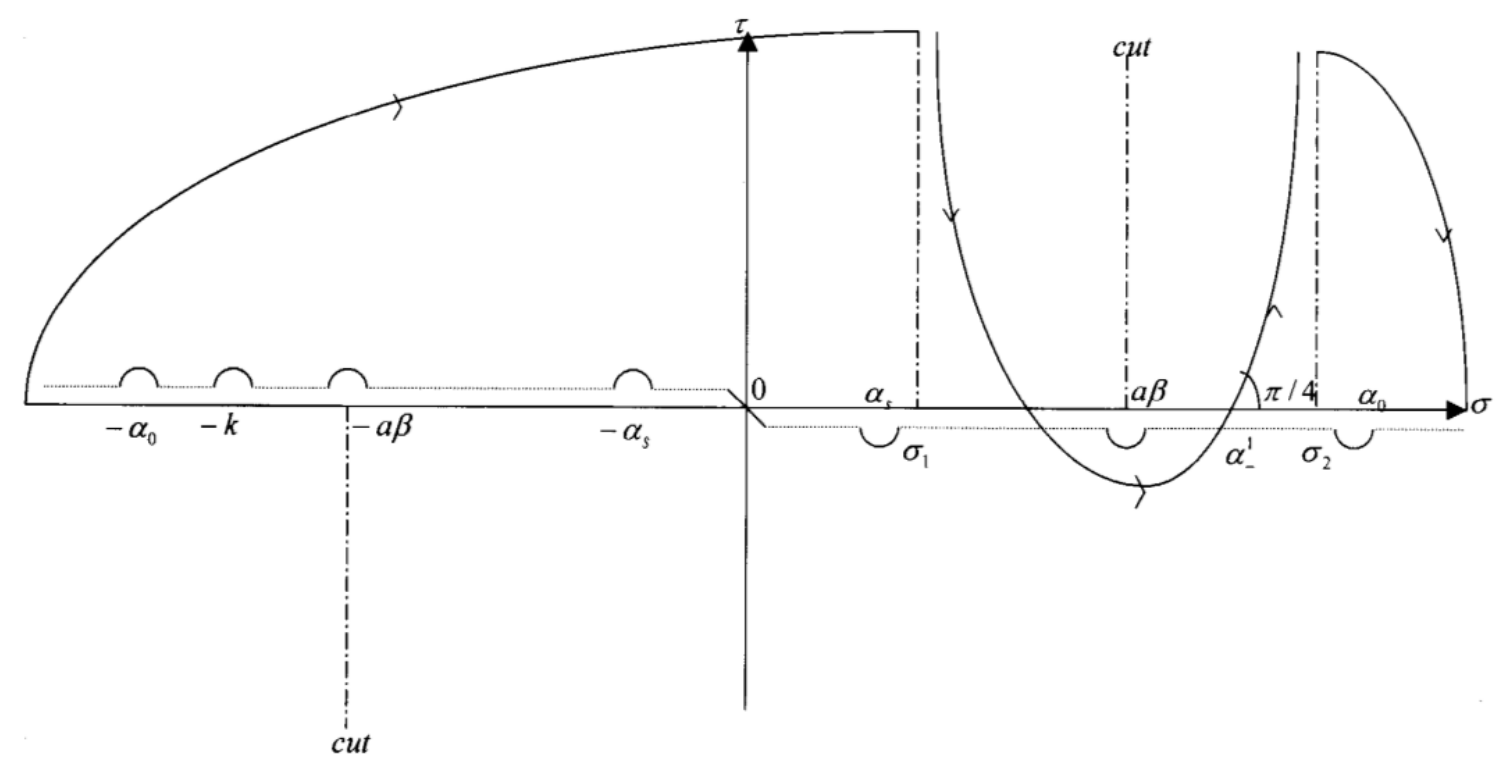

Fig.3. Representation of contour passing through the saddle point in $(\pi / 2, \pi)$.

From Figs 2 and 3, it is clear that the integrals in Eq.(3.48) involving the poles $\alpha= \pm \alpha_{s}$ for $\omega_{s}<\omega<\omega_{0}$ (i.e., in the domain $\left.(x<0) \bigcup(x>l)\right)$ and the poles $\alpha= \pm \alpha_{0}$ for $\omega_{p}<\omega<\omega_{0} \quad$ (i.e., $0<x<l$ ) are to be evaluated by using the residue theorem and denoted by $\varphi_{s}$ and $\phi_{E S}$, respectively.

Thus 


$$
\begin{aligned}
& \phi_{s}=\frac{\Omega_{l}\left(\alpha_{s}\right) \exp \left[\frac{\beta}{1+a^{2}}\left\{\left(a^{2}-1\right) y-2 i a^{2} x\right\}\right]}{\left\{\gamma\left(\alpha_{s}\right)-i a \beta\right\} \Omega_{2}^{\prime}\left(\alpha_{s}\right)}\left[\frac{i H K^{+}(k)}{\alpha_{s}+k}+\left(\frac{\omega^{2}}{g}\right)^{1 / 2} Q\left(\alpha_{s}\right)\right]+ \\
& -\left(\frac{\omega^{2}}{g}\right)^{1 / 2} \frac{\Omega_{l}\left(\alpha_{s}\right) R\left(-\alpha_{s}\right)}{\left\{\gamma\left(-\alpha_{s}\right)-i a \beta\right\} \Omega_{3}^{\prime}\left(-\alpha_{s}\right)} \exp \left[\frac{\beta}{1+a^{2}}\left\{\left(a^{2}-1\right) y+2 i a^{2}(x-l)\right\}\right]
\end{aligned}
$$

and

$$
\begin{aligned}
& \left.\left.\phi_{E S}=\frac{\Omega_{l}\left(-\alpha_{0}\right) \exp \left[\frac{\beta\left(1+a^{2}\right) c-2 g \rho_{0}}{\left(1+a^{2}\right) c} y+i \alpha_{0} x\right]}{\left\{\gamma\left(-\alpha_{0}\right)-i a \beta\right\} \Omega_{2}^{\prime}\left(-\alpha_{0}\right)}\right] \frac{i H K^{+}(k)}{k-\alpha_{0}}+\left(\frac{\omega^{2}}{g}\right)^{1 / 2} Q\left(-\alpha_{0}\right)\right]+ \\
& -\left(\frac{\omega^{2}}{g}\right)^{l / 2}\left[\frac{\Omega_{l}\left(-\alpha_{0}\right) R\left(\alpha_{0}\right)}{\left\{\gamma\left(\alpha_{0}\right)-i a \beta\right\} \Omega_{3}^{\prime}\left(\alpha_{0}\right)} \exp \left[\frac{\beta\left(1+a^{2}\right) c-2 g \rho_{0}}{\left(1+a^{2}\right) c} y-i \alpha_{0}(x-l)\right]+\right. \\
& +\frac{\Omega_{l}\left(-\alpha_{1}\right) R\left(\alpha_{1}\right)}{\left\{\gamma\left(\alpha_{1}\right)-i a \beta\right\} \Omega_{3}^{\prime}\left(\alpha_{1}\right)} \exp \left[\frac{i \gamma\left(\alpha_{1}\right) y}{a}-i \alpha_{l}(x-l)\right]+ \\
& \left.+\frac{\Omega_{l}\left(-\alpha_{2}\right) R\left(\alpha_{2}\right)}{\left\{\gamma\left(\alpha_{2}\right)-i a \beta\right\} \Omega_{3}^{\prime}\left(\alpha_{2}\right)} \exp \left[\frac{i \gamma\left(\alpha_{2}\right) y}{a}-i \alpha_{2}(x-l)\right]\right]
\end{aligned}
$$

Thus we see that $\phi_{s}$ is the combination of two waves of different amplitudes traveling away from the edges of the strip in different directions. Also $\phi_{S}$ is called the surface wave which is localized near the free surface and exists only for $\omega_{s}<\omega<\omega_{0}$.

Again from Eq.(4.12), we conclude that $\phi_{E S}$ is the sum of two waves, of different amplitudes, which exist in the region under the strip and after superposition, it forms a Kelvin wave, which is localized near the inertial strip for $\omega_{p}<\omega<\omega_{0}$.

When the contour passes through the saddle point $\alpha_{n}^{2}$, we can determine $\phi_{n}^{2}$ similarly. The term $\phi_{n}^{2}$ arises in the region $\theta_{c}<\theta_{n}<\pi-\theta_{c}$, due to diffraction, i.e., in the outside region of the characteristic cone. However, unlike $\phi_{n}^{l}$, it has no wave-like character and it decays exponentially away from the edge of the strip in the region $\theta_{c}<\theta_{n}<\pi-\theta_{c}$.

Thus

$$
\phi_{n}^{2} \approx \chi_{l}\left(\alpha_{n}^{2}\right) \frac{a \beta \sin \theta_{n}}{\Psi^{3 / 2}\left(\theta_{n}\right)} \sqrt{\frac{2 \pi}{\beta r_{n}}} \exp \left\{-r_{n} \beta \Psi\left(\theta_{n}\right)+\frac{i \pi}{2}\right\}+O\left(\frac{1}{\beta r_{n}}\right) .
$$

Hence the final result for the total wave field $\Theta$ is obtained in the following form

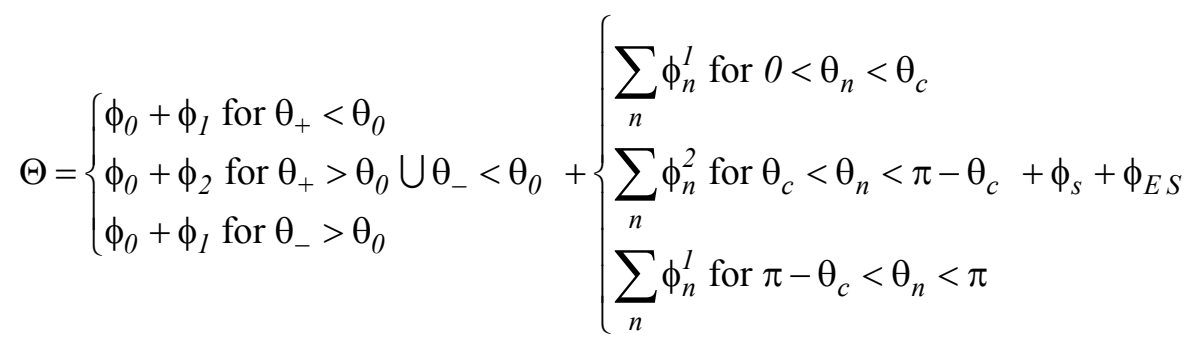


Here $\phi_{2}$ is the wave reflected from the elastic plate. From the representation of $\phi_{2}$, it is clear that if we make rigidity of the elastic plate to tend to infinity and the surface density to tend to zero, then $\phi_{2}$ becomes a wave reflected from a solid wall. Also from relation (2.9), if $k^{2}=\left(\frac{\sigma_{1}}{\lambda}\right)^{1 / 2} \omega$, then we have $\phi_{2}=\phi_{1}$. In this case $\phi_{n}^{1}, \phi_{n}^{2}, \phi_{S}, \phi_{E S}$ all vanish, which correspond to the absence of diffraction.

For $k^{2} \neq\left(\frac{\sigma_{l}}{\lambda}\right)^{l / 2} \omega, \phi_{n}^{l}$ represents the diffracted field in the region of the characteristic cone, i.e., $\theta_{n} \in\left[\left(0, \theta_{c}\right) \bigcup\left(\pi-\theta_{c}, \pi\right)\right]$. From the representation of $\theta_{n}^{l}$, it may be noted that the amplitude of the waves decays as $\left(\beta r_{n}\right)^{-1 / 2}$. The crests of these waves form a family of hyperbola with common asymptotes which coincide with the boundaries of the characteristic cones $\theta=\theta_{c}$ and $\theta=\pi-\theta_{c}$.

The term $\phi_{n}^{2}$ represents the diffracted field outside of the characteristic cones, i.e., $\theta_{n} \in\left(\theta_{c}, \pi-\theta_{c}\right)$. From the representation of $\phi_{n}^{2}$, it is also clear that the diffracted field in this region does not have a wave like character and it decays exponentially away from the edges of the strip in the region $\theta_{c}<\theta_{n}<\pi-\theta_{c}$. The term $\phi_{S}$ denotes the combination of two waves of different amplitudes traveling away from the edges of the strip in different directions, and $\phi_{E S}$ is the sum of two waves, also of different amplitudes, which exist in the region under the strip. Also we see that the integrand of Eq.(3.48) has four complex poles at the points $\pm \alpha_{1}$ and $\pm \alpha_{2}$ situated in the upper and lower half planes, respectively. We note that the residues of the integrand at the complex poles $-\alpha_{1},-\alpha_{2}$ decay exponentially on moving away from the left end of the strip and from the residues of the poles $\alpha_{1}, \alpha_{2}$, we also note that in the presence of the second edge of the elastic strip two progressive waves propagate in the region of the right side characteristic cone.

\section{Conclusion}

In this paper, the problem of internal wave diffraction by a strip of an elastic plate present on the surface of an exponentially stratified liquid is investigated. The problem is formulated in terms of a function related to the stream function and the associated boundary value problem involves the Klein-Gordon equation. In the mathematical analysis, we use the approximate Wiener-Hopf technique for a large width of the strip and the diffracted field is obtained in terms of integrals, which are evaluated asymptotically in different regions for a large distance from the edges of the strip. The characteristic equation of the KleinGordon equation represents a pair of straight lines, which forms two characteristic cones with centers at the two ends of the strip respectively. Again, we see that the diffracted field has wave like character in the region of the characteristic cones, but outside the characteristic cones it has no wave like character. We also note that the wave generated due to the presence of the strip is at higher frequencies than the wave generated on the free surface of the liquid. Also we conclude that two progressive waves propagate in the region of the characteristic cone lying on the right side.

\section{Nomenclature}

$$
\begin{aligned}
l & - \text { length of the elastic plate } \\
p & - \text { dynamic pressure } \\
R & - \text { reflection coefficient } \\
t & - \text { time } \\
v_{x}-x & - \text { component of velocity }
\end{aligned}
$$




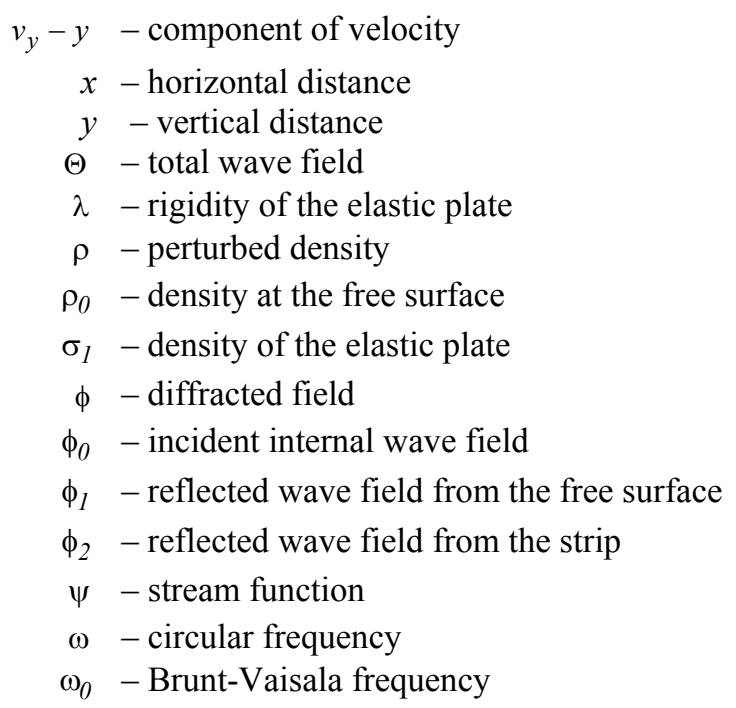

\section{Acknowledgement}

I thank Dr. B. N. Mandal of the Indian Statistical Institute, Kolkata, for his supervision in the preparation of the paper.

\section{References}

Dolai P. and Mandal B.N. (2007): Scattering of internal waves in a stratified fluid by the edge of an inertial surface.Bull. Cal. Math. Soc., vol.99, pp.5-20.

Gabov S.A. and Svesnikov A.G. (1982): On the diffraction of internal waves by the edge of an ice field. - Soviet. Math. Dokl., vol.26, pp.8-11.

Gayen R., Mandal B.N. and Chakrabarti A. (2007): Water wave diffraction by a surface strip. - J. Fluid Mech., vol.571, pp.419-438.

Gayen R., Mandal B.N. and Chakrabarti A. (2006): Water wave scattering by two sharp discontinuities in the surface boundary conditions. - IMA J. Appl. Math., vol.71, pp.811-831.

Kanoria M., Mandal B.N. and Chakrabarti A. (1999): The Wiener-Hopf solution of a class of mixed boundary value problems arising in surface water wave phenomena. - Wave Motion, vol.29, pp.267-292.

Noble B. (1958): Methods based on the Wiener-Hopf technique for the solution of partial differential equations. - New York: Pergamon Press

Varlamov V.V. (1983): Diffraction of internal waves in a stratified liquid on a semi-infinite wall. - U.S.S.R. Comput. Maths. Math. Phys., vol.23, pp.87-91.

Varlamov V.V. (1985): The scattering of internal waves by the periphery of an elastic plate. - U.S.S.R. Comput. Maths. Math. Phys., vol.25, pp.58-63.

Received: January 7, 2012

Revised: August 2, 2012 